\title{
Integral treatment of teaching waves
}

\author{
Lia Zerbino, N. Baade, M. Lavagna, A. Mocoroa
}

Lia Maria Zerbino, N. N. Baade, M. E. Lavagna, A. B. Mocoroa, "Integral treatment of teaching waves," Proc. SPIE 3831, Sixth International Conference on Education and Training in Optics and Photonics, (16 June 2000); doi: 10.1117/12.388701 


\title{
Integral treatment in the Waves Teaching
}

\author{
L. M. Zerbino(1) (4) (5), N. N. Baade(2) (3) M. E. Lavagna (2) (3) \& A. B. Mocoroa(3) \\ IMApEC \& OPTIMO \\ Facultad de Ingeniería, Universidad Nacional de La Plata, Argentina \\ E-mail: liaz@odin.ciop.unlp.edu.ar
}

Keywords: Curricula, integration of concepts, waves teaching, models, analogies, basic university courses.

\begin{abstract}
Using like basic idea that the similar equations corresponds to the similar models and they can explain similar phenomena with characteristic own, a strategy has been developed for the integrated treatment of the waves teaching.

The analogies pointed out by the mathematical equations allow unifying topics like that of the propagation of the electromagnetic energy, the propagation of the energy through the matter and the mechanic-quantum treatment of the systems evolution. The wealth of the analysis of the meanings of the wave functions as well as of the coefficients involved in the equations allows to carry out a conceptual synthesis of the natural phenomenology.

Results of the application of this strategy in basic university courses are commented.
\end{abstract}

\section{INTRODUCTION}

The need of satisfying formation requirements of young generations of professionals, preparing them to assimilate current and future developments in science and technology, requires the educational system to be organized so as to ensure that learners achieve, in a reasonably short period of time, the conceptualization needed to be able to understand and assimilate these developments.

In the case of Physics nowadays, many of those advancements are related to the interaction between radiation and matter. Its comprehension involves, undoubtedly, the need to analyze in depth and integrate the partial and limited conceptualization that leads to an independent study of the different topics included in traditional curricula.

General Physics courses at our universities usually present a traditional succession of chapters inducing the generation of quasi-still compartments to present the contents, often leaving on a second plane fundamental principles and integrating concepts which are the real milestones of our theories, and the basis of the mechanisms that led to their formulation. This not only hinders the global formation of the physical world, but also - and even worse - conceals the real essence of scientific thinking, whose transference is one of the fundamental objectives of the teaching of basic sciences, thus reducing the efficacy of the application of constructivistic methodologies.

Pedagogues have been making a clear choice towards constructivistic methods for a long time now, as the most genuine way of achieving meaningful learning. To build up a conceptual background based on previous knowledge, by reelaborating, associating and correlating, in order to arrive to new concepts, seems to be the best guarantee to achieve our objective.

In an attempt of putting these characteristics into practice, we tried the simultaneous and parallel presentation of different topics in grade courses of Engineering, highlighting similarities and differences. Taking as a starting point the idea that similar equations correspond to similar models, and that they can explain similar phenomena with their own characteristics, we have developed strategies for an integrated teaching of different topics that admit the same physical model [1]

These are the basis for a curricular proposal, elaborated according to topics integration to achieve a unified learning, seeking topics integration and highlighting the characteristics that different situations have in common. This proposal was presented 
for the PUFFAL project, UNESCO[2]. This methodology was implemented in workshops for the formation of teachers[3], and in some regular courses of General Physics. The advantages of this method were that not only it allowed the integration of concepts, but it also presented a considerable increase in efficiency in the sense that we were able to "teach more in less time".

As an extension of this same approach, in order of not only integrating previous knowledge but also in an attempt of "leaving the doors open" for the treatment of topics that are developed further on in other courses, we have tried to integrate a brief comment as regards probability waves to the global treatment of mechanic and electromagnetic waves, previously implemented [3]. This early presentation of a topic that will be dealt with further on in the course of studies - Quantum Mechanics - is intended only to pre-announce this conceptualization and strengthen the idea that the concept of wave involves, in addition to the simple classical case, more complex cases where the interaction with the environment produces cushioning, resonance or non-linear phenomena.

\section{OUTLINE OF THE PRESENTATION}

Figure 1 presents a scheme to show the outline for the simultaneous teaching of the topics mechanic waves, electromagnetic waves, and an introduction to the concept of probability waves. The special characteristics of each phenomena under study are analyzed: propagation, properties of the media, transported magnitude, boundary conditions.

As it can be inferred from this outline, once the simplifications needed to get the homogeneous classical wave equation are carried out, and the characteristics of the solutions for the cases of electromagnetic and elastic waves are found, the De Broglie postulates are mentioned, and the Schrödinger equation, whose solution is called Schrödinger wave function or probability amplitude function, is presented. The idea is to confront this equation with the classical wave equation in order to discuss the wave character - or not - of the solutions. Thus, it becomes evident that the Schrödinger wave equation has been given this name because its resolution is a wave somewhat similar to the wave equation of classical theory, although the similarity is not close.

In differential classical equations for mechanics and electromagnetism, there appear second derivatives with respect to space and second derivatives with respect to time (so far the homogeneous wave equation). There may also appear first derivatives with respect to time with real coefficients, and sources. In Schrödinger's differential equation for quantum mechanics, there are second derivatives with respect to space, but not with respect to time. Also, the term with a first derivative with respect to time has an imaginary coefficient.

That is, considering the case without sources, we have, both for mechanics and each of the components of the electromagnetic field, in absence of conductors,

$\nabla^{2} \Phi(\overrightarrow{\mathrm{r}}, \mathrm{t})=\frac{1}{\mathrm{v}^{2}} \frac{\partial^{2} \Phi(\overrightarrow{\mathrm{r}}, \mathrm{t})}{\partial \mathrm{t}^{2}}$

while if there is loss or diffusion we have

$\nabla^{2} \Phi(\vec{r}, \mathrm{t})=\frac{1}{v^{2}} \frac{\partial^{2} \Phi(\vec{r}, \mathrm{t})}{\partial \mathrm{t}^{2}}+D \frac{\partial \Phi(\overrightarrow{\mathrm{r}}, \mathrm{t})}{\partial \mathrm{t}}$

In both equations, the speed $\mathbf{v}$ is characterised by the system properties (elastic modules and densities in the mechanic case, permissiveness and permeability in the electromagnetic case).

In the first equation, if there is a linear relationship between the wave number and the frequency, the solution must be of the type $\Phi(\vec{r}, t)=f(\vec{k} \cdot \vec{r}-\omega t)$ with $f$ real, an wave solution is obtained.

In the second equation, such linearity is not present, if $\mathrm{D}$ is real it adds a cushioning of the form $e^{-a t}$ to the solution, the result being a cushioned solution. 


\begin{tabular}{|c|c|c|}
\hline \multicolumn{3}{|c|}{ Wave equations including the time } \\
\hline \multicolumn{2}{|r|}{ Previous Concepts } & New Concepts \\
\hline $\begin{array}{l}\text { Stress, Pressure, } \\
\text { Strain }\end{array}$ & $\begin{array}{l}\text { Electromagnetic Fields } \\
\text { Maxwell's equations }\end{array}$ & $\begin{array}{l}\text { Wave-Particle Duality } \\
\text { De Broglie }\end{array}$ \\
\hline \multicolumn{2}{|r|}{ Classic Physics } & Quantum Mechanics \\
\hline Elastic Waves & Electromagnetic Waves & Probability Waves \\
\hline & $\begin{array}{l}\text { E and B general equations } \\
\Gamma: \mathrm{E}(r, t)=\mu \varepsilon \frac{\partial^{2} \mathrm{E}(r, t)}{\partial t^{2}}+\mu \sigma \frac{\partial \mathrm{E}(r, t)}{\partial t}+\nabla \cdot\left(\frac{\rho}{\varepsilon}\right) \\
\Gamma: \overrightarrow{\mathbf{B}}_{(r, t)}=\mu \varepsilon \frac{\partial^{2} \overrightarrow{\mathbf{B}}_{(r, t)}}{\partial t^{2}}+\mu \sigma \frac{\partial \overrightarrow{\mathbf{B}}_{(r, t)}}{\partial t}\end{array}$ & \multirow{3}{*}{$\begin{array}{l}\text { Particle of mass } \mathrm{m} \text { in a } \\
\text { scalar potential field } \mathrm{V}(\mathrm{r}) \text {. } \\
\text { In this case, De Broglie } \\
\text { relations } \\
\lambda=\frac{h}{p} ; \quad v=\frac{\varepsilon}{h}\end{array}$} \\
\hline 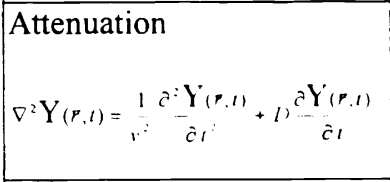 & 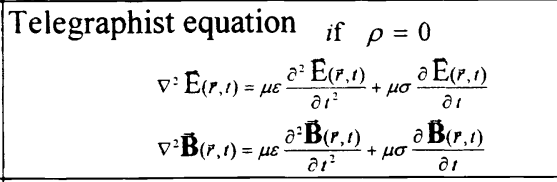 & \\
\hline $\begin{array}{l}\text { Mechanic wave equation } \\
\text { if } D=0 \\
\nabla^{2} Y(p, t)=\frac{1}{v} \hat{c}_{v}^{:} \frac{Y(p, t)}{\partial t^{2}}\end{array}$ & $\begin{array}{c}\text { Electromagnetic wave equations } \\
\text { if } \sigma=0 \text { and if } \rho=0 \\
\nabla^{2} \overrightarrow{\mathrm{E}}(\vec{r}, t)=\mu \varepsilon \frac{\partial^{2} \mathrm{E}(\vec{r}, t)}{\partial t^{2}} \\
\nabla^{2} \vec{B}(\vec{r}, t)=\mu \varepsilon \frac{\partial^{2} \vec{B}_{(\vec{r}, t)}}{\partial t^{2}}\end{array}$ & \\
\hline \multicolumn{3}{|c|}{$\nabla: \Phi(\vec{r}, t)=\frac{1}{v^{2}} \frac{\partial^{2} \Phi_{(\vec{r}, t)}}{\partial t^{2}} ; \quad \Phi(\overrightarrow{\mathrm{r}}, \mathrm{t})$ real function } \\
\hline \multicolumn{3}{|c|}{ Were $v$ represents the system } \\
\hline$v=\begin{array}{l}\text { Longitudinal ; } \\
T \\
\mu\end{array} \quad v=\bar{\beta}$ & Transversal $v=\frac{1}{\sqrt{\varepsilon \mu}}$ & \\
\hline Necessar & $k^{2}=\frac{\omega^{2}}{v^{2}}$ & $\mathrm{k}^{2}={ }^{2 \mathrm{~m}}(\omega-\mathrm{V}(\overrightarrow{\mathrm{r}}))=\stackrel{\mathrm{p}}{ }$ \\
\hline $\begin{array}{l}\text { General wave equation } \\
\qquad \begin{array}{r}\nabla^{2} \\
\Psi\end{array}\end{array}$ & $\begin{array}{l}\Psi(\vec{r}, t)=\frac{k^{2}}{\omega^{2}} \frac{\partial^{2} \Psi(r, t)}{\partial t^{2}} \\
(P, t) \text { complex function }\end{array}$ & $\begin{array}{l}\text { Schröedinger equation } \\
\qquad \begin{aligned}-\frac{\hbar^{2}}{2 m} \nabla^{2} \Psi(\vec{r}, t)+V(\vec{r}) \Psi(\vec{r}, t)=i \hbar \frac{\partial \Psi(\vec{r}, t)}{\partial t} \\
\\
\Psi(\vec{r}, t) \text { complex function }\end{aligned}\end{array}$ \\
\hline$\Phi(r . t)=f(k \cdot$, & $-\omega t)$ or $\Phi(\vec{r}, t)=\operatorname{Re} \Psi_{0} e^{i(\vec{k} \bullet p-\omega t)}$ & $\Psi(\vec{r}, t)=\Psi_{0} e^{i(\vec{k} \bullet r-\omega t)}$ \\
\hline
\end{tabular}




\begin{tabular}{|c|c|c|}
\hline \multicolumn{3}{|c|}{ Since t does not appear explicitly in the equations, I may try variables separation } \\
\hline \multicolumn{3}{|c|}{$\widetilde{\Psi}(\vec{r}, t)=\widetilde{R}(\vec{r}) \cdot \widetilde{T}(t)$} \\
\hline & $\begin{array}{l}\nabla^{2} \widetilde{R}(\vec{r})+k^{2} \widetilde{R}(\vec{r})=0 \\
\frac{d^{2} \widetilde{T}(t)}{d t^{2}}+v^{2} k^{2} \widetilde{T}(t)=0\end{array}$ & $\begin{array}{l}\nabla^{2} \widetilde{R}(\vec{r})+\frac{2 m}{\hbar^{2}}[\varepsilon-V(\vec{r})] \widetilde{R}(\vec{r})=0 \\
\frac{d \widetilde{T}(t)}{d t}+\frac{i}{\hbar} \varepsilon \widetilde{T}(t)=0\end{array}$ \\
\hline $\begin{array}{l}\text { 1) } \\
\text { 2) } \\
\text { 3) } \\
\text { 4) }\end{array}$ & $\begin{array}{l}\text { Environment conditions determine certain values of } \mathrm{k} \\
\widetilde{T}(t) \propto e^{-i \omega_{n} t} \\
\widetilde{\Psi}_{n}(\vec{r}, t)=\widetilde{R}_{n}(\vec{r}) \cdot \widetilde{T}_{n}(t) \text { characterises state } \mathrm{n} \\
\widetilde{\Psi}(\vec{r}, t)=\sum_{n} C_{n} \widetilde{\Psi}_{n}(\vec{r}, t) \text { general solution }\end{array}$ & $\begin{array}{l}\text { 1) solutions that work only for certain values } \\
\text { of } \varepsilon_{n} \text { (self values), then for each } \varepsilon_{n} \text { there } \\
\text { is an } \widetilde{R}_{n}(\vec{r}) \text { (self function) } \\
\text { 2) } \widetilde{T}(t) \propto e^{-\frac{t}{\hbar} \varepsilon_{n} t} \\
\text { 3) } \Psi_{n}(\vec{r}, t)=\widetilde{R}_{n}(\vec{r}) \cdot \widetilde{T}_{n}(t) \text { characterises } \\
\text { state } \mathrm{n} \\
\text { 4) } \widetilde{\Psi}(\vec{r}, t)=\sum_{n} C_{n} \widetilde{\Psi}_{n}(\vec{r}, t) \text { general solution }\end{array}$ \\
\hline
\end{tabular}

Figure 1

In Schrödinger's differential equation for quantum mechanics, there is a term with second derivatives with respect to space, a term with a first derivative with respect to time, an imaginary coefficient, and the term with a second derivative with respect to time is missing

$-\frac{\hbar^{2}}{2 \mathrm{~m}} \nabla^{2} \widetilde{\Psi}(\overrightarrow{\mathrm{r}}, \mathrm{t})+\mathrm{V}(\overrightarrow{\mathrm{r}}) \widetilde{\Psi}(\overrightarrow{\mathrm{r}}, \mathrm{t})=\mathrm{i} \hbar \frac{\partial \widetilde{\Psi}(\overrightarrow{\mathrm{r}}, \mathrm{t})}{\partial \mathrm{t}}$

Even though there are no second derivatives with respect to time, the existence of an imaginary diffusion coefficient i $\hbar$ makes the solution a complex function with wave behaviour.

When temporal and spatial variables are separated, which is not a completely crazy idea since there is no explicit time component in differential equations, the same expression is obtained for spatial differential equations, whereas for temporal variables the expressions are different.

In addition to this, in the case of mechanic and electromagnetic waves, it is mentioned that the solutions must be real because they correspond to measurable physical magnitudes, while in the case of quantum mechanics, the solution is complex, and what has physical meaning is $|\psi|^{2}$, which is interpreted as a probability density. Thus, the denomination of probability wave functions is partly justified for the solutions of this equation, despite the fact that Schrödinger's equation cannot be considered, in a strict classical sense, a wave equation.

\section{CONCLUSIONS}

This presentation, tested in a workshop for teachers, allowed to analyse in detail the physical concepts involved. Participants looked for and discussed the mathematical and physical arguments for the different developments, and were motivated to differentiate the consequences that the particular physical conditions - both of the media and the intervening magnitudes imposed upon the solutions found. Even though the presentation of fundamental ideas of quantum mechanics was only introductory, it set the basis for a future treatment where the traditionally adopted nomenclature will not lead to the frequent conceptual conflicts arising when the denominations used to indicate similarities - but not taking into account differences are taken word for word. 


\section{REFERENCES}

1. Unificación de temas en base a similitudes y diferencias, presented at the Simposio de Investigadores en Educación en Física, SIEF, Facultad de Ciencias Exactas y tecnológicas Universidad Nacional de Tucumán (UTN), Tucumán, Argentina, 1992.

2. Propuesta curricular para la unificación de temas en Física. PUFFAL project, UNESCO, Rio de Janeiro, Brazil, LatinAmerican Meeting for the evaluation of the project 1993.

3. Resistencias, capacitancias y bobinas nos muestran las similitudes y diferencias en el campo electromagnético. workshop given at the REF9, Salta, 1995)

4. Ondas mecánicas y electromagnéticas, workshop for teachers, Escuela Integrada de Física de Olavarría, Olavarría, Argentina, 1995.

\section{BIBLIOGRAPHY}

1. E. Mezbacher, Quantum Mechanics, John Wiley \& sons, inc. New York-London, 1961

2. I. Peral Alonso, Ecuaciones en derivadas parciales, Addison-Wesley-Universidad Autónoma de Madrid, 1995.

3. A. Pipes, Applied Mathematics for engineers and physicist, Mc Graw-Hill, 1958.

4. M. Born \& E. Wolf, Principles of Optics, Pergamon Press

5. E. Hech y A. Zajac, Optica, Fondo Educativo Interamericano, 1986. 